\title{
Acute Triangulations of Trapezoids and Pentagons
}

\author{
Maddalena Cavicchioli \\ Department of Economics, University of Venice “Cá Foscari”, San Giobbe, Cannaregio 873, 30121 Venice, Italy
}

Correspondence should be addressed to Maddalena Cavicchioli; maddalena.cavicchioli@unive.it

Received 20 November 2012; Accepted 6 February 2013

Academic Editor: Antonio Gómez-Corral

Copyright (C) 2013 Maddalena Cavicchioli. This is an open access article distributed under the Creative Commons Attribution License, which permits unrestricted use, distribution, and reproduction in any medium, provided the original work is properly cited.

An acute triangulation of a polygon is a triangulation whose triangles have all their angles less than $\pi / 2$. The number of triangles in a triangulation is called the size of it. In this paper, we investigate acute triangulations of trapezoids and convex pentagons and prove new results about such triangulations with minimum size. This completes and improves in some cases the results obtained in two papers of Yuan (2010).

\section{Introduction and Preliminaries}

A triangulation of a planar polygon is a finite set of nonoverlapping triangles covering the polygon in such a way that any two distinct triangles are either disjoint or intersect in a single common vertex or edge. An acute (resp., nonobtuse) triangulation of a polygon is a triangulation whose triangles have all their angles less (resp., not larger) than $\pi / 2$. The number of triangles in a triangulation is called the size. Burago and Zalgaller [1] and, independently, Goldberg and Manheimer [2] proved that every obtuse triangle can be triangulated into seven acute triangles and this bound is the best possible. Cassidy and Lord [3] showed that every square can be triangulated into eight acute triangles and eight is the minimum number. This remains true for any rectangle as proved by Hangan et al. in [4]. Acute triangulations of trapezoids, quadrilaterals, and pentagons were investigated in [5-8]. Further information, historical notes, and problems about acute triangulations of polygons and surfaces can be found in the survey paper [9]. Let $\mathscr{K}$ denote a family of planar polygons, and for $K \in \mathscr{K}$, let $f(K)$ be the minimum size of an acute triangulation of $K$. Then, let $f(\mathscr{K})$ denote the maximum value of $f(K)$ for all $K \in \mathscr{K}$. The following results are known.

Theorem 1. (i) Reference [7]: let $\mathscr{T}$ denote the family of all trapezoids, that is, quadrilaterals with at least one pair of parallel sides. Then, $f(\mathscr{T} \backslash \mathscr{R})=7$, where $\mathscr{R}$ is the family of all rectangles (also including squares).

(ii) Reference [6]: let $Q$ be the family of all quadrilaterals. Then, $f(\mathbb{Q})=10$.

(iii) Reference [5]: let $\widehat{Q}_{c}$ be the family of all convex quadrilaterals. Then, $f\left(\mathbb{Q}_{c}\right)=8$.

(iv) Reference [8]: let $\mathscr{P}$ denote the family of all planar pentagons. Then, $f(\mathscr{P}) \leq 54$.

In this paper, we discuss acute triangulations of trapezoids and convex pentagons and prove new results of such triangulations with minimum size. For example, we get the following characterization of the right trapezoids: they are the only trapezoids needing exactly six triangles and one interior vertex for an acute triangulation of minimum size. For the family of convex pentagons, we show that the bound stated in Theorem 1(iv) can be improved under some additional conditions.

Let $\Gamma$ be a convex planar polygon. A vertex $P$ of $\Gamma$ is called an acute (resp., right) corner if the interior angle of $\Gamma$ at $P$ is less than (resp., equal to) $\pi / 2$; otherwise, $P$ is called an obtuse corner. Let $\mathbf{T}$ be an acute triangulation of $\Gamma$. A vertex or edge of $\mathbf{T}$ is called a boundary (resp., interior) vertex or edge if it lies on the boundary of $\Gamma$ (resp., lies inside $\Gamma$ ). Let $n, n_{i}$, and $n_{\partial}$ be the number of vertices, interior vertices, and boundary vertices of $\mathbf{T}$. Let $m, m_{i}$, and $m_{\partial}$ be the number of edges, interior edges, and boundary edges of $\mathbf{T}$. Clearly, we 
have $n=n_{i}+n_{\partial}, m=m_{i}+m_{\partial}$, and $n_{\partial}=m_{\partial}$. For each vertex $P$ in $\mathrm{T}$, the number of edges incident to $P$ is called the degree of $P$, denoted by $\operatorname{deg}(P)$. Let $v_{k}$ denote the number of vertices in $\mathbf{T}$ of degree $k$. Let $f$ denote the number of triangles in $\mathbf{T}$. The following lemma is easily verified (cf. [6, Lemma 1]).

Lemma 2. Let $\mathbf{T}$ be an acute triangulation of a planar convex $n$-gon $\Gamma$. Then, one has

(1) $f=m-n+1=n+n_{i}-2=m_{i}-n_{i}+1$,

(2) $3 f=2 m-m_{\partial}=2 m_{i}+n_{\partial}$,

(3) $v_{1}=0 ; v_{2}+v_{3} \leq n$,

(4) $n_{\partial}=m_{\partial} \leq f+v_{2} ; 4 f+v_{2} \geq 2 m$,

(5) If a vertex $P$ of $\mathbf{T}$ is an interior vertex, then $\operatorname{deg}(P) \geq 5$, if $P$ lies within a side of $\Gamma$, then $\operatorname{deg}(P) \geq 4$, and if $P$ is an obtuse or right corner of $\Gamma$, then $\operatorname{deg}(P) \geq 3$.

\section{Characterizations of Trapezoids}

2.1. Parallelograms. Let $A B C D$ be a parallelogram with acute corners at $B$ and $D$ and $|A B| \leq|B C|$. If the diagonal $A C$ divides the angles at $A$ and $C$ into acute angles, then $A B C D$ is triangulable with exactly two acute triangles. Otherwise, we have the following lemma that completes Theorem 2.1 in Section 2 of [7].

Lemma 3. Let $A B C D$ be a parallelogram with acute corners at $B$ and D. Suppose that the diagonal $A C$ does not divide the angle at $A$ (or $C$ ) into acute angles. Then, $A B C D$ is triangulable with four acute triangles, and this bound is the best possible.

Proof. Let $f$ be the smallest number of triangles in an acute triangulation $\mathbf{T}$ of the parallelogram $A B C D$. Some edge of $\mathbf{T}$ incident with $A$ (resp., $C$ ) must meet the interior of $A B C D$. Denote $A P$ to be such an edge, and suppose that $P$ is an interior vertex of $A B C D$, that is, $n_{i} \geq 1$. Then, the vertices $B$ and $D$ have degree at least 2 , the vertices $A$ and $C$ have degree at least 3 , and the vertex $P$ has degree at least 5 . Summing up, we have $2 m \geq 2 \times 2+2 \times 3+5=15$, hence $2 m \geq 16$. Since $v_{2} \leq 2$, by Lemma 2(4), we get $4 f+2 \geq 16$, so $f \geq 4$. Assume now that $n_{i}=0$. Then, $P$ lies in the interior of the edge $B C$ (or $D C)$. Otherwise, if $P=C$, at least one of the vertices $A$ and $C$ must have degree $\geq 4$ by hypothesis. This implies that there is a neighbor of it with degree $\geq 4$, giving a contradiction as $n_{i}=0$. Thus, $n_{\partial}=n$ and $m_{i} \geq 3$ as the degree of $P$ is at least 4 , and the degree of $A$ (resp., $C$ ) is at least 3. By Lemma 2(1), we get $f=m_{i}-n_{i}+1 \geq 4$. Now, for a parallelogram as in the statement, an acute triangulation with 4 triangles is given in [7, Section 2].

2.2. Trapezoids. Following [7], we say that a trapezoid is a quadrilateral with at least one pair of parallel sides. Let $A B C D$ be a trapezoid with parallel sides $A D$ and $B C$ with $|A D|<$ $|B C|$. Let $E$ (resp., $F$ ) be the orthogonal projection of $A$ (resp., $D)$ on the straight line $\ell_{B C}$ containing $B C$. Suppose that $E$ is interior to $B C$ and $F$ exterior to $B C$. If the diagonal $A C$ divides the angles at $A$ and $C$ into acute angles, then $A B C D$ is triangulable with exactly two acute triangles. Otherwise, we have the following lemma which can be proved in the same manner as Lemma 3.

Lemma 4. Let $A B C D$ be a trapezoid with acute corners at $B$ and $D$, parallel sides $A D$ and $B C$ with an obtuse angle $B \hat{A} C$, and an acute angle $C \hat{A} D$. Then, $A B C D$ is triangulable with four acute triangles, and this bound is the best possible.

Let $A B C D$ be a trapezoid with two adjacent acute angles at $B$ and $C$, parallel sides $A D$ and $B C$, and, consequently, $|A D|<|B C|$. If there exists an interior point $P$ on $B C$ such that the triangles $B A P, A P D$, and $P D C$ are acute, then $A B C D$ is triangulable by 3 acute triangles. Otherwise, we have the following lemma.

Lemma 5. Let $A B C D$ be a trapezoid with two adjacent acute angles at $B$ and $C$ and parallel sides $A D$ and $B C$. Suppose that there is no interior point $P$ on $B C$ such that $B A P, A P D$, and $P D C$ are acute triangles. Then, $A B C D$ is triangulable with five acute triangles, and this bound is the best possible.

Proof. Let $f$ be the smallest number of triangles in an acute triangulation $\mathbf{T}$ of any trapezoid as in the statement. Some edge $A P$ must meet the interior of $A B C D$. Suppose that $P$ is an interior point of $A B C D$, that is, $n_{i} \geq 1$. Then, it immediately follows that $\operatorname{deg}(P) \geq 5$ and so $f \geq 5$. Suppose that there is no interior vertex in $A B C D$, that is, $n_{i}=0$ and $P$ is a boundary vertex. Some edge $D Q$ must meet the interior of $A B C D$, and $Q$ is also a boundary vertex. We can assume that $P \neq Q$ since at least one of the triangles $B A P, A P D$, and $P D C$ is not acute by hypothesis. This implies that $m_{i} \geq 4$. By Lemma 2(1), $f=m_{i}-n_{i}+1 \geq 5$. Now, for a trapezoid as in the statement, an acute triangulation with 5 triangles is given in $[7$, Section 3].

The following result gives a characterization of the right trapezoids.

Proposition 6. Every trapezoid with exactly two right angles is triangulable with six acute triangles and one interior vertex, and this bound is the best possible.

Proof. Let $f$ be the smallest number of triangles in an acute triangulation $\mathbf{T}$ of any trapezoid $A B C D$ with exactly two right corners at $D$ and $C$. Let $B$ be an acute corner, thus $|A B| \leq|B C|$. Some edge $A P$ must meet the interior of $A B C D$. Suppose that $P$ is an interior vertex of $A B C D$ and $n_{i}=1$. Then, $P$ is an end vertex of five interior edges. At least one neighbour of $P$ is interior to a side of the trapezoid and is therefore incident of a further interior edge. So, $m_{i} \geq 6, n_{i}=1$ and $f=m_{i}-n_{i}+1 \geq 6$. Suppose that $P$ is an interior vertex and $n_{i} \geq 2$. Then, there are at least two interior vertices with degree $\geq 5$, the degree of $A$ (resp., $C$ and $D$ ) is $\geq 3$, the degree of $B$ is $\geq 2$, and there is at least one vertex of degree $\geq 4$. Then, we have $2 m \geq 3 \times 3+2 \times 5+4+2 \geq 25$, so $2 m \geq 26$. Since $v_{2} \leq 1$ by Lemma 2(4), we get $4 f+1 \geq 26$, so $f>6$. Suppose that $n_{i}=0$ and $n_{\partial}=n$. Some edge of AP must meet the interior of $A B C D$. Then $P$ cannot lie on the edge $D C$; otherwise, some edge incident to $D$ must meet the interior of $A B C D$, and we 
get an interior vertex against the fact that $n_{i}=0$. So, $P$ must be in the interior of $B C$. If the angles at $P$ are not right, we get a contradiction since $A P C D$ is a trapezoid with only two right angles which admits an acute triangulation of at least size $f$. But this contrasts with the minimum size $f$ of $\mathbf{T}$. If the angles at $P$ are right, then $A P C D$ is a square. But any acute triangulation of a square must have at least an interior vertex. So, we get again a contradiction as $n_{i}=0$. Now, for a trapezoid as in the statement, an acute triangulation with 6 triangles and one interior vertex are given in [7, Section 3].

Lemma 7. Let $A B C D$ be a trapezoid with acute corners at $B$ and $D$, parallel sides $A D$ and $B C$, and $E$ and $F$ both exterior to $B C$. Then, $A B C D$ is triangulable with seven acute triangles. This bound is the best possible among the acute triangulations of such a trapezoid which have at least one interior vertex.

Proof. Let $\mathbf{T}$ be an acute triangulation with at least one interior vertex for a trapezoid as in the statement. Let $f$ denote the size of $\mathbf{T}$. Suppose that $n_{i}=2$. Then, there are two interior vertices $P$ and $S$ in $\mathbf{T}$, and at least two neighbours of $P$ and/or $S$ have degree at least 4 . Since $m_{i} \geq 9$ and $n_{i}=2$, Lemma 2(1) gives $f=m_{i}-n_{i}+1 \geq 8$. Suppose that $n_{i} \geq 3$. Then, there are at least three interior vertices $P, R$, and $S$ with degree $\geq 5$. The degrees of $A$ and $C$ are $\geq 3$, and those of $B$ and $D$ are $\geq 2$. There are at least two neighbours of $P$, $R$ and/or $S$ with degree $\geq 4$. Summing up, we have $2 m \geq$ $3 \times 5+2 \times 3+2 \times 2+2 \times 4=33$, hence $2 m \geq 34$. Since $v_{2} \leq 2$, by Lemma 2(4), we get $4 f+2 \geq 34$, hence $f \geq 8$. So, we can assume that $n_{i}=1$. The interior vertex $P$ cannot be connected to all the vertices of $A B C D$. Otherwise, there is a neighbour of $P$ which is interior to a side of the trapezoid. It is therefore adjacent to a further interior vertex of the trapezoid, that is, $n_{i} \geq 2$. This contradicts $n_{i}=1$. If $P$ is connected to exactly three vertices of the trapezoid, say $A, B$, and $C$, there are two neighbours of $P$ which have degree $\geq 4$. Further, at least one of the obtuse corners $A$ and $C$ must have degree $\geq 4$. Then, we have $m_{i} \geq 7, m_{\partial}=n_{\partial}=n-1$ and $n+f-1=m_{i}+m_{\partial} \geq 7+n-1$, hence $f \geq 7$. If $P$ is joined to exactly two vertices of $A B C D$, then there are three neighbours of $P$ with degree $\geq 4$. The degree of the two vertices of $A B C D$ joined with $P$ is $\geq 3$, and the remaining two vertices have degree $\geq 2$. Summing up, we get $2 m \geq 5+2 \times 2+2 \times 3+3 \times 4=27$, hence $2 m \geq 28$. Since $v_{2} \leq 2$, by Lemma 2(4), we have $4 f+2 \geq 28$, hence $f \geq 7$. If $P$ is joined to exactly one vertex of $A B C D$, then four neighbours of $P$ have degree $\geq 4$. Thus, $m_{i} \geq 7$, and, by Lemma 2(1), $f=m_{i}-n_{i}+1 \geq 7$. Now, for a trapezoid as in statement, an acute triangulation with 7 acute triangles was described in [7, Section 3].

\section{Acute Triangulations of Pentagons}

The following proposition follows directly from the results proved in [5].

Proposition 8. Every convex quadrilateral admits an acute triangulation of size at most eight, such that there are at most two new vertices introduced on each side.
It was shown in $[8$, Lemma 3.1] that every pentagon with at least one acute corner can be triangulated into at most 32 acute triangles. Under the hypothesis of convexity, we have the following.

Proposition 9. Every convex pentagon with at least one acute corner can be triangulated into at most 25 acute triangles.

Proof. Let $\Gamma=A B C D E$ (in the anticlockwise order) be a convex pentagon with at least one acute corner, say $A$. We distinguish some cases.

Case 1. The triangle $A B E$ is acute. By Proposition 8 , the convex quadrilateral $B C D E$ has an acute triangulation with size $\leq 8$ such that there are at most 2 side vertices on $B E$.

Subcase 1.1. There is no side vertex on BE. Then, $\Gamma$ admits an acute triangulation with at most 9 triangles.

Subcase 1.2. There is precisely one side vertex, say $P$, on $B E$. By Lemma 4 of [6], since $A B E$ is an acute triangle, for any point $P$ on the side $B E$, there are two points $R$ on $A E$ and $S$ on $A B$ such that the line segments $P R, R S$, and $P S$ divide $A B E$ into four acute triangles. Then, we get an acute triangulation of $\Gamma$ into at most 12 triangles.

Subcase 1.3. There are exactly two side vertices, say $M$ and $N$, on $B E$. In this case, the convex quadrilateral $B C D E$ has an acute triangulation of size 7, as shown in [5]. Suppose that $M$ is an interior point of $E N$. Let $P$ be the point on $A E$ such that $P N$ is parallel to $A B$. Then, the triangle $P N E$ is acute. By Lemma 4 of [6], the triangle $P N E$ can be triangulated into four acute triangles with $M$ as the only side vertex on $E N$ and two new vertices $R$ and $S$ on the edges $E P$ and $N P$, respectively. Let $H$ and $K$ be the orthogonal projections of $S$ and $N$ on the edge $A B$. The segments $N K$ and $S K$ divide the right trapezoid $H B N S$ into three right triangles. By [7, Section $3]$, there is an acute triangulation of the right trapezoid AHSP of size 6 without new vertices introduced on the sides $P S$ and $H S$. Then, we can slightly slide $K$ and $H$ in direction from $B$ to $A$ such that the triangles $S H K, S K N$, and $B K N$ become acute. This gives an acute triangulation of $\Gamma$ whose size is at most 20.

Case 2. The triangle $A B E$ is nonacute, that is, $E$, for example, is a nonacute corner.

Subcase 2.1. There is no side vertex on $B E$. Then, there exists an acute triangle, say $E B P$, which belongs to an acute triangulation of $B C D E$ with size $\leq 8$. Let $H$ be the orthogonal projection of $P$ on the side $B E$. By Theorem 2 of [6], since $A B E$ is a triangle with nonacute corner $E$, for any point $H$ on the side $B E$, there is an acute triangulation of $A B E$ with size 7 such that $H$ is the only side vertex lying on $B E$. Such an acute triangulation of $A B E$ has new vertices $R$ (resp., $S$ and $T$ ) introduced on the side $A E$ (resp., $A B$ ). Finally, we can slightly slide $H$ away from $E B P$ in direction perpendicular to $H P$ such that the triangles EHP and $B H P$ become acute. This gives an acute triangulation of $\Gamma$ into at most 16 acute triangles.

Subcase 2.2. There is precisely one side vertex $P$ on $B E$. As in the previous subcase, by Theorem 2 of [6], for any point $P$ 
on the side $B E$, there is an acute triangulation of the triangle $A B E$ with size 7 such that $P$ is the only side vertex lying on $B E$. This gives an acute triangulation of $\Gamma$ into at most 15 acute triangles.

Subcase 2.3. There are exactly two side vertices, say $M$ and $N$, on $B E$. In this case, $B C D E$ has an acute triangulation of size 7 by [5]. Suppose that $M$ is an interior point of $E N$. Let $P$ be the point on $A E$ such that $P N$ is parallel to $A B$. Then, the triangle $E N P$ has a nonacute corner $E$. By Theorem 2 of [6], ENP can be triangulated into 7 acute triangles with $M$ as the only side vertex on $E N$ and new vertices $R$, respectively, $S$ and $T$ on the edges $E P$, respectively, $N P$. Let $H, K$, and $L$ be the orthogonal projections of $S, T$, and $N$ on the edge $A B$, respectively. The line segments $N L, T L, T K$, and $S K$ divide the right trapezoid HBNS into 5 right triangles. By [7, Section 3], there is an acute triangulation of the right trapezoid AHSP of size 6 without new vertices introduced on the sides $P S$ and $H S$. Then, we can slightly slide $L, K$, and $H$ in direction from $B$ to $A$ such that the triangles $B L N, T N L, K T L, K T S$, and $S H K$ become acute. This gives an acute triangulation of $\Gamma$ whose size is at most 25.

Corollary 10. Every convex pentagon with at least two nonadjacent acute corners can be triangulated into at most 16 acute triangles.

Proof. By the hypothesis and the results from [5], we can avoid subcases 1.3 and 2.3 in the above proof. The remaining cases give the requested bound.

The following proposition follows directly from the results proved in $[3,4,7]$.

Proposition 11. Every trapezoid (resp., rectangle) admits an acute triangulation of size at most 7 (resp., 8) such that there are at most one new vertex introduced on each side.

Proposition 12. Let $\Gamma$ be a convex pentagon which has at least one acute corner and two parallel sides, nonincident to it. Then, $\Gamma$ can be triangulated into at most 14 acute triangles.

Proof. Let $\Gamma=A B C D E$ (in the anticlockwise order) be a convex pentagon with at least one acute corner, say $D$, and two parallel sides $A E$ and $B C$ with $|A E| \leq|B C|$. We distinguish some cases.

Case 1. The triangle CDE is acute.

Subcase 1.1. Let $H$ and $K$ be the orthogonal projections of $A$ and $E$, respectively, on the straight line $\ell_{B C}$ containing the edge $B C$. Suppose that $H$ (resp., $K$ ) is interior (resp., exterior) of $B C$. By [7, Section 2], the trapezoid $A B C E$ admits an acute triangulation of size at most 4 such that there are no new vertices on the side $C E$. Then, $\Gamma$ has an acute triangulation of size at most 5 .

Subcase 1.2. Suppose that the above orthogonal projections $H$ and $K$ are interior to the edge $B C$. By [7, Section 2], the trapezoid $A B C E$ admits an acute triangulation of size at most 5 such that no new vertices are introduced on $C E$. Then, $\Gamma$ has an acute triangulation of size at most 6 .
Subcase 1.3. Suppose that $A B C E$ is a right trapezoid (this implies that the triangle $C D E$ is acute). By [7, Section 3], $A B C E$ can be triangulated into 6 acute triangles such that there are no new vertices on $C E$. Then, $\Gamma$ has an acute triangulation of size at most 7.

Subcase 1.4. Suppose that the above orthogonal projections $H$ and $K$ are exterior to $B C$. By [7, Section 3], $A B C E$ admits an acute triangulation of size at most 7 such that there is only one vertex, say $P$, on the side $C E$. By Lemma 4 of [6], for any point $P$ in the side $C E$, there are two points $R$ on $D E$ and $S$ on $C D$ such that the line segments $P R, P S$, and $R S$ divide $C D E$ into 4 acute triangles. Then, $\Gamma$ can be triangulated into at most 11 acute triangles.

Case 2. The triangle $C D E$ is nonacute, that is, the corner $E$, for example, is nonacute.

Subcase 2.1. Let $A B C E$ be as in subcase 1.1. There is an acute triangle, say $C E P$, which belongs to the triangulation of size $\leq 4$ of $A B C E$. Let $R$ be the orthogonal projection of $P$ on the edge $C E$. By Theorem 2 of [6], there is an acute triangulation of $C D E$ with size 7 such that $R$ is the only side vertex on $C E$. Then, $\Gamma$ has an acute triangulation of size at most 12 .

Subcase 2.2. Let $A B C E$ be as in subcase 1.2. Reasoning as in the previous subcase gives an acute triangulation of $\Gamma$ with size $\leq 13$.

Subcase 2.3. Let $A B C E$ be as in Subcase 1.4. By Theorem 2 of [6] the triangle $C D E$ can be triangulated into at most 7 acute triangles such that the only side vertex on $C E$ is $P$. This gives an acute triangulation of $\Gamma$ with size $\leq 14$.

\section{Acknowledgment}

The author would like to thank the anonymous referees for their careful reading and very useful comments and suggestions.

\section{References}

[1] Y. D. Burago and V. A. Zalgaller, "Polyhedral embedding of a net," Vestnik St. Petersburg University, vol. 15, pp. 66-80, 1960 (Russian).

[2] M. Goldberg and W. Manheimer, "Elementary Problems and Solutions: Solutions: E1406," The American Mathematical Monthly, vol. 67, no. 9, p. 923, 1960.

[3] C. Cassidy and G. Lord, "A square acutely triangulated," Journal of Recreational Mathematics, vol. 13, no. 4, pp. 263-268, 1980/81.

[4] T. Hangan, J.-I. Itoh, and T. Zamfirescu, "Acute triangulations," Bulletin Mathématique de la Société des Sciences Mathématiques de Roumanie, vol. 43(91), no. 3-4, pp. 279-285, 2000.

[5] M. Cavicchioli, "Acute triangulations of convex quadrilaterals," Discrete Applied Mathematics, vol. 160, no. 7-8, pp. 1253-1256, 2012.

[6] H. Maehara, "On acute triangulations of quadrilaterals," in Discrete and Computational Geometry (Tokyo, 2000), vol. 2098 of Lecture Notes in Comput. Sci., pp. 237-243, Springer, Berlin, Germany, 2001.

[7] L. Yuan, "Acute triangulations of trapezoids," Discrete Applied Mathematics, vol. 158, no. 10, pp. 1121-1125, 2010. 
[8] L. Yuan, "Acute triangulations of pentagons," Bulletin Mathématique de la Société des Sciences Mathématiques de Roumanie, vol. 53(101), no. 4, pp. 393-410, 2010.

[9] T. Zamfirescu, "Acute triangulations: a short survey," in Proceedings of the VI Annual Conference of the Romanian Society of Mathematical Sciences, Vol. I (Romanian) (Sibiu, 2002), pp. 10-18, Soc. Ştiinţ e Mat. România, Bucharest, 2003. 


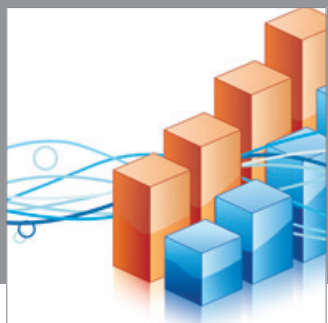

Advances in

Operations Research

mansans

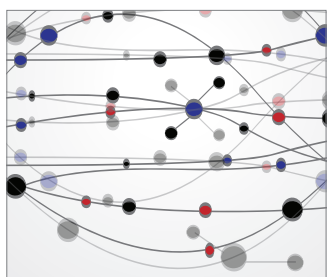

The Scientific World Journal
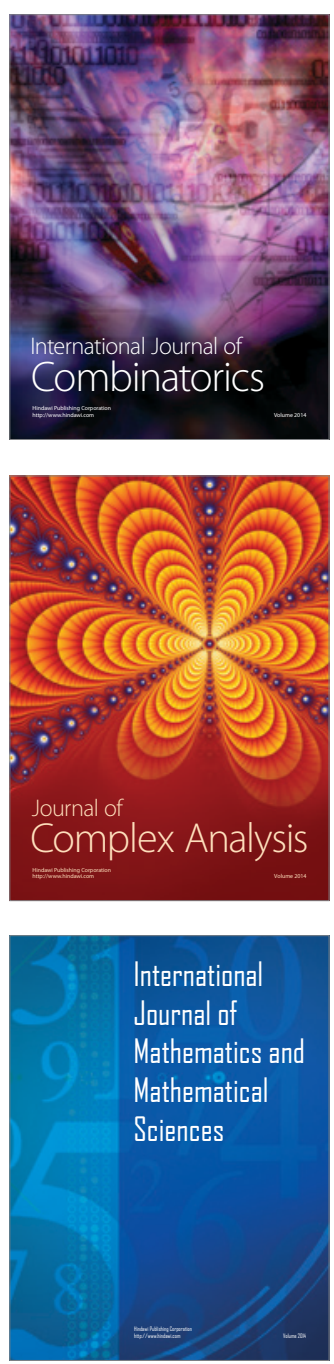
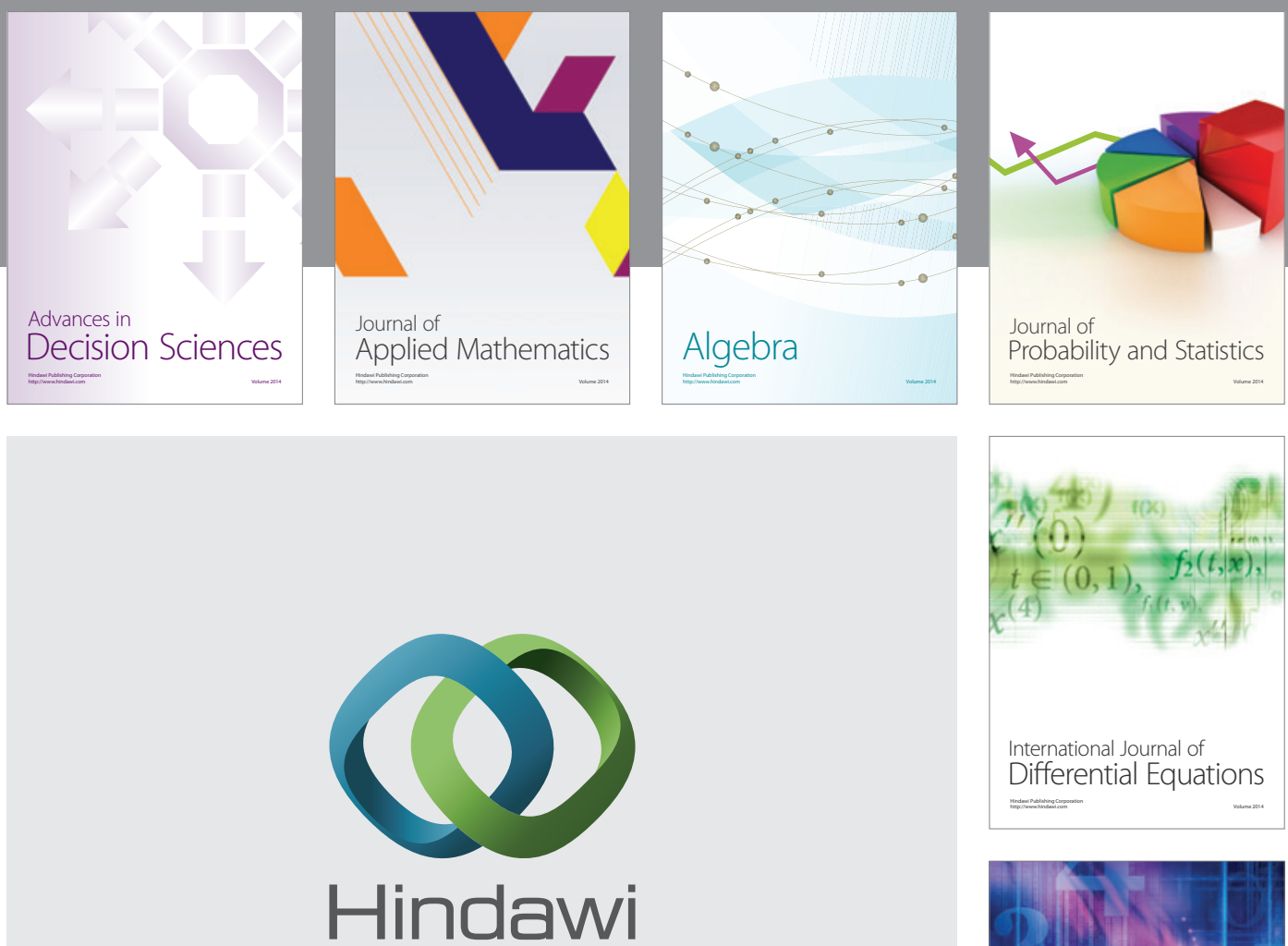

Submit your manuscripts at http://www.hindawi.com
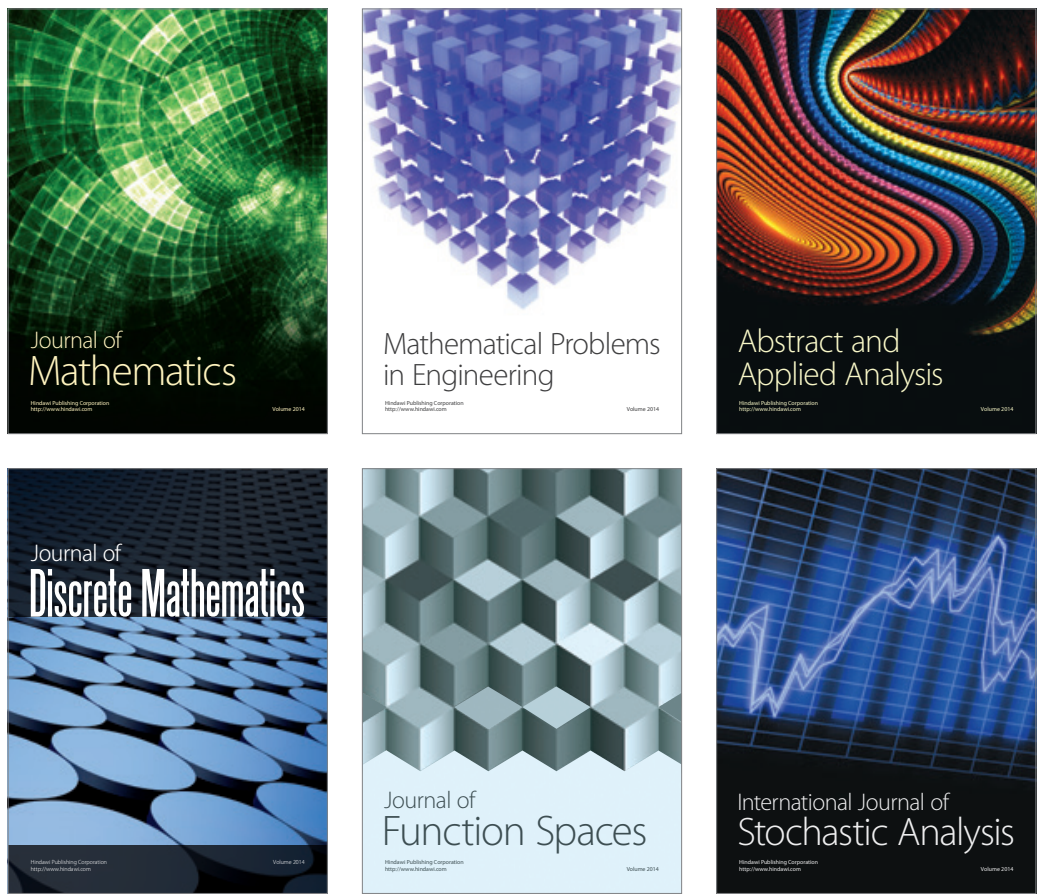

Journal of

Function Spaces

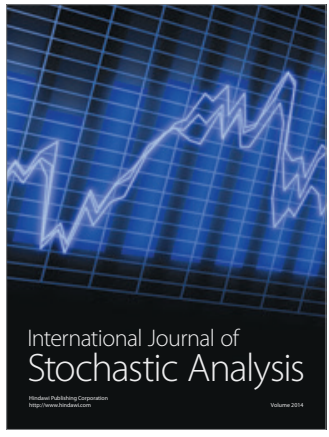

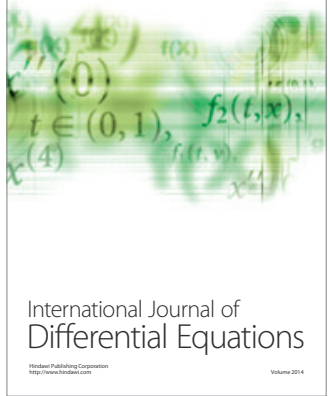
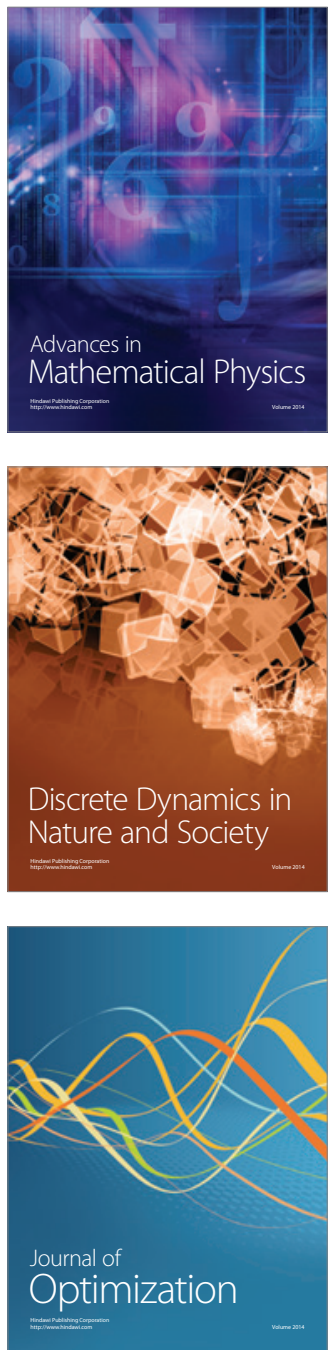